\title{
Tras las huellas de la investigación en educación de la física desde los proyectos del IDEP, el CIUP y la Universidad Distrital Francisco José de Caldas en la década 1995-2005 \\ Following tracks of the investigation in physical science education from the researches projects of IDEP, CIUP and Universidad Distrital Francisco José de \\ Caldas during 1995-2005 decade.
}

Jaime D. Reyes R. ${ }^{1}$

\begin{abstract}
Resumen
En este artículo se presenta una organización de los trabajos de investigación que se desarrollaron en la década 1995-2005 entorno a la enseñanza y el aprendizaje de la física en Bogotá, a partir de categorías que surgen como resultado de la sistematización de información documental en el marco del desarrollo de antecedentes y estado del arte del proyecto de tesis doctoral Conocimiento Didáctico del Contenido del profesor de Fisica en formación inicial, el caso de la enseñanza del campo eléctrico. Forma parte del apartado sobre investigaciones en Colombia, particularmemnte en Bogotá, que se reportaron en el IDEP, el CIUP y la Universidad Distrital en el campo de la enseñanza y el aprendizaje de la física. La documentación fue analizada a partir de la base de datos que el autor y un grupo de investigadores construyeron previamente en el proyecto de investigación avalado por el CIDC (Reyes, J., 2007).
\end{abstract}

\section{Palabras clave:}

Investigación en educación, ciencias, física, enseñanza, aprendizaje, tendencias.

\begin{abstract}
This article presents an organization of the researches developed in the 1995-2005 decade about teaching and learning physics in Bogota. The categories used in this work arise as a result of the systematization of documentary information and take part of the development of doctoral thesis titled pedagogical content knowledge of pre-service physics teacher about teaching electric field. The papers used here were reported by the Instituto para la Investigación Educativa y el Desarrollo Pedagógico IDEP, the
\end{abstract}

1 Profesor Universidad Distrital Francisco José de Caldas. Magister Universidad Pedagógica Nacional, Maestría en Docencia de La Física. Correo electrónico: jreyesr@udistrital.edu.co 
Centro de Investigaciones de la Universidad Pedagógica Nacional CIUP and the Universidad Distrital FJC, in the field of teaching and learning of physics. The documentation was analyzed from the database that the author and a research team previously built in the research project supported by the Centro de Investigación y Desarrollo Científico CIDC (Reyes, J., 2007).

\section{Key words}

Investigation in Education, Sciences, Physics, Education, Learning, Tendencies.

\section{Introducción}

El estudio de la enseñanza y el aprendizaje de las ciencias tiene diversas perspectivas; por un lado, en los programas de formación de educadores de ciencias -física, química, biología- se asumen algunas líneas de aproximacion investigativa, estilo monografías; a su vez en los programas de maestría en educación o en docencia de las ciencias, se hace lo propio con las tésis de grado e igualmente, en los últimos años, en el marco de los programas de doctorado en educación en ciencias.

De otra parte, se encuentran los trabajos de investigación institucionalizados con pretensiones específicas en la enseñanza y el aprendizaje de las ciencias, inscritos oficialmente en las bases de datos de universidades, entidades gubernamentales o instituciones públicas o privadas y que se identifican en una categoría genérica como lo es la de "trabajo de investigación". Es en este marco donde surge la investigación de la cual se presentan aquí algunos resultados en el campo de la enseñanza y aprendizaje de la física, de acuerdo con una primera caracterización de los trabajos encontrados en educación en ciencias en Colombia en la década 1995-2005 en la Universidad Pedagógica de Colombia, de la Universidad Distrital "Francisco José de Caldas" y del IDEP.

La revisión de las investigaciones y publicaciones sobre educación en ciencias debe formar parte del acumulado de conocimiento actualmente en Co- lombia. De hecho, existe un compendio, resultado de investigación, de trabajos en el campo de la enseñanza de las ciencias (Hernández C. 2002) en el cual se hace referencia a investigaciones apoyadas por Colciencias y algunos textos consecuencia de tales investigaciones. Hernández distingue algunos elementos característicos del campo de la educación en ciencias en Colombia desde la perspectiva de la enseñanza a saber:

1. Las ideas previas, preteorías o preconceptos de los estudiantes, 2. Constructivismo, el aprendizaje significativo y la enseñanza por problemas, 3. Precisiones conceptuales y construcción o estudio de teorías sobre el aprendizaje de conceptos o sobre la relación pedagógica ,4. Otras investigaciones trabajan sobre los docentes, sobre sus creencias sobre la ciencia y sobre el quehacer del educador, 5. Algunas investigaciones se interesan explícitamente en el modo como contribuye el conocimiento de las ciencias o, mejor aún, la apropiación de la cultura científica en la formación integral de los estudiantes, 6 . Otras investigaciones se ocupan de la relación entre la tecnología y el aprendizaje de las ciencias, 7. También se realiza un tipo de investigación pedagógica que conduce a la producción de textos o de medios para la enseñanza y 8. Una línea de investigación que resulta cada vez más importante es la que explora diferencias entre distintas situaciones escolares, entre distintos contextos culturales, entre distintas estrategias pedagógicas, entre distintas formas de organización de los contenidos o entre distintas formas de aprender (2002).

En este trabajo se estructura una forma de organizar la investigación en educación en ciencias, desde la perspectiva principal de los trabajos de investigación financiados por Colciencias, pero no se agrupan aquellas investigaciones con fuentes de financiación diferentes como universidades, instituciones educativas o institutos. De igual forma, no se presenta una tendencia de los trabajos de investigación en el campo de la enseñanza y el aprendizaje de la física específicamente. 


\section{Metodología}

El enfoque metodológico de este trabajo tiene sus fundamentos en la investigación documental de carácter descriptivo (Ortiz, 1993), desarrollada por medio de los procesos de consecución de la información y validez de las fuentes, la lectura analítica de resúmenes y contenidos, en caso de ser necesario, y la estructuración de una base de datos de consulta especializada.

Los documentos que se constituyen como fuentes, en esta investigación, son los informes y productos de investigación en educación en ciencias en Colombia en la década 1995-2005, que han sido organizados en el marco de una investigación desarrollada previamente por el autor y otros investigadores, de la cual se generó una base de datos y un compendio de material físico y en medio magnético objeto del análisis particular que se presenta en este artículo. De esta forma, la investigación se define documental en el sentido de la interpretación primaria de productos comunicativos, como infor-

\begin{tabular}{|c|c|c|c|c|c|}
\hline \multicolumn{6}{|c|}{$\begin{array}{l}\text { TABLA 1. Principales tendencias de proyectos } \\
\text { De investigación en educación y ciencias } \\
\text { en Colombia 1995- } 2005\end{array}$} \\
\hline & Norte & \begin{tabular}{|c|} 
Sur \\
Occidente
\end{tabular} & \begin{tabular}{c|c|} 
Nor \\
Occidente
\end{tabular} & Centro & \begin{tabular}{|l|} 
Total \\
\end{tabular} \\
\hline Curriculo & 0,61 & 1,82 & 1,82 & 4,87 & 9,1 \\
\hline $\begin{array}{l}\text { Naturaleza del } \\
\text { Conocimiento }\end{array}$ & 1,22 & 1,22 & 0,61 & 10,37 & 13,4 \\
\hline Profesión Docente & 1,22 & 0 & 3,66 & 9,76 & 14,6 \\
\hline Trabajo Experimental & 0 & 0,61 & 1,22 & 4,88 & 6,7 \\
\hline Enseñanza - & 0,61 & 1,22 & 4,27 & 26,2 & 32,3 \\
\hline $\begin{array}{c}\text { Resolución de } \\
\text { Problemas }\end{array}$ & 0 & 0,61 & 0,61 & 1,22 & 2,4 \\
\hline TICs & 0 & 1,83 & 0,61 & 2,44 & 4,9 \\
\hline Historia & 0 & 0,61 & 0 & 0,61 & 1,22 \\
\hline CTS Medio Ambiente & 0 & 0 & 0 & 9,76 & 9,7 \\
\hline Otras & 0,61 & 0 & 1,83 & 3,66 & 6,1 \\
\hline $\begin{array}{l}\text { Fuente: Reyes J. (2 } \\
\text { Investigación En Echuca } \\
\text { Final Proyecto de Inv } \\
\text { Caldas" CIDC. Grupos } \\
\text { 2403520305 }\end{array}$ & $\begin{array}{l}\text { 7) Anál } \\
\text { on En Cl } \\
\text { tigación } \\
\text { de inves }\end{array}$ & $\begin{array}{l}\text { isis Epistem } \\
\text { iencias En Ch } \\
\text { Universidad } \\
\text { tigación: Inv }\end{array}$ & $\begin{array}{l}\text { nológico } Y \\
\text { nolombia } 199 \\
\text { Distrital "F } \\
\text { vaucol - Int }\end{array}$ & $\begin{array}{l}\text { Cultural } \\
5-2005 . \\
\text { rancisco } \\
\text { tercitec. }\end{array}$ & $\begin{array}{l}\text { De La } \\
\text { Informe } \\
\text { José De } \\
\text { Código: }\end{array}$ \\
\hline
\end{tabular}

mes de investigación y resultados de los mismos, y se enmarca en un primer nivel de los procesos de análisis de contenido (Gaitán 1998) entendiendo este como conocimiento "guardado" en forma de documento y que se hace necesario extraer en virtud de los propósitos específicos del investigador.

\section{Resultados y análisis: tendencias generales en investigación en educación en ciencias en la década 1995 - 2005 en Colombia}

Como resultado del trabajo documental realizado se encontraron 164 proyectos de investigación en educación en ciencias en la década mencionada. De acuerdo con la tabla 1, en esta década se hace énfasis mayoritariamente en la investigación sobre procesos de enseñanza-aprendizaje, un 32,3\% de los trabajos manifiestan esta tendencia en forma más significativa. En segundo lugar, se encuentra que la profesión docente es objeto de estudio en un $14,6 \%$ de las investigaciones, seguida por los estudios sobre la naturaleza del conocimiento con un $13,4 \%$ de las investigaciones estudiadas. Ahora bien, aspectos como ciencia, tecnología, sociedad y ambiente con un 9,7\% y estudios sobre el currículo en ciencias con un 9,1\% también forman parte de los intereses de los investigadores y en un menor grado se encuentran el trabajo experimental $(6,7 \%)$, las TICs $(4,9 \%)$, la resolución de problemas $(2,4 \%)$ y los estudios de corte histórico-epistemológico $(1,22 \%)$.

En general, puede afirmarse que la investigación en educación en ciencias en Colombia en la década estudiada, a la luz de los trabajos de investigación consultados, revela una preocupación por los procesos tanto de aprendizaje de las mismas por parte de los estudiantes como de la enseñanza por parte de los profesores, caracterizada principalmente por establecer causas posibles de ser abordadas, lo cual se refleja en el interés por plantear alternativas frente a problemáticas detectadas, dándole una relativa importancia a categorías de investigación asociadas a la naturaleza del conocimiento científico, a la Tecnología de Información y Comunicación y a la tendencia CTS. Al parecer, otro interés se asocia a la necesidad de estudiar y reformar el currículo de ciencias en la estructura educativa, 
aspecto que se manifiesta como relevante en un grupo de trabajos.

Si bien debe reconocerse que este tipo de inferencias requieren un nivel de contrastación más profundo en la medida en que se puedan relacionar, específicamente con los trabajos de investigación consultados, conviene mantenerlos como un referente general para el propósito principal de este documento, del cual se ocupa la siguiente sección y que pretende ahondar en la clasificación de los trabajos de investigación específicamente en el campo de la educación en la física

La mayoría de las investigaciones son lideradas por las universidades públicas y privadas, en segundo lugar se encuentran los colegios y otras instituciones. Ahora bien, al igual que en la tendencia global de investigación en educación en ciencias en Colombia, la zona centro lidera tanto en número como en diversidad de propuestas de investigación en educación en la física.

\section{Tendencias generales en investigación en educación en física 1995 -2005}

La resolución de problemas, como campo de investigación, manifiesta el mayor porcentaje de trabajos, aspecto que sugiere una perspectiva de orden más pragmático que de contribución al corpus teórico de la educación en la física. Los trabajos agrupados en esta categoría en general consideran relevante la formulación de problemas que propician la aplicación de conocimiento físico y que a su vez favorezcan su recontextualización. Un análisis más profundo de cada uno de estos trabajos permitiría su caracterización de acuerdo con los intereses de los grupos de investigación.

La profesión docente, en el caso de la enseñanza de la física, se encuentra en el segundo lugar de interés de los investigadores, ya sea en el caso de la formación inicial de profesores, como en el de los procesos de actualización de formación permanente de profesores de física en ejercicio.

El trabajo experimental comparte el interés de los grupos de investigación. Esta tendencia busca va- lidar la importancia de los trabajos prácticos desde perspectivas alternativas que van más allá de la simple organización de guías o instrucciones, y propende por vincular la idea de experimento a los procesos de enseñanza de acuerdo con intereses de los estudiantes.

Tanto el currículo como la naturaleza del conocimiento físico también son de interés para los grupos de investigación, en las primeras se aprecia la preocupación por contextualizar la enseñanza de la física desde perspectivas culturales así como cualificar las competencias científicas de los estudiantes a partir de los lineamientos del M.E.N. Algunas de estas investigaciones se complementan con aquellas que procuran validar los estudios históricos como referente en el diseño de propuestas didácticas.

Las investigaciones que explicitan el doble paradigma enseñanza-aprendizaje plantean la necesidad de revisar y proponer formas más eficientes en
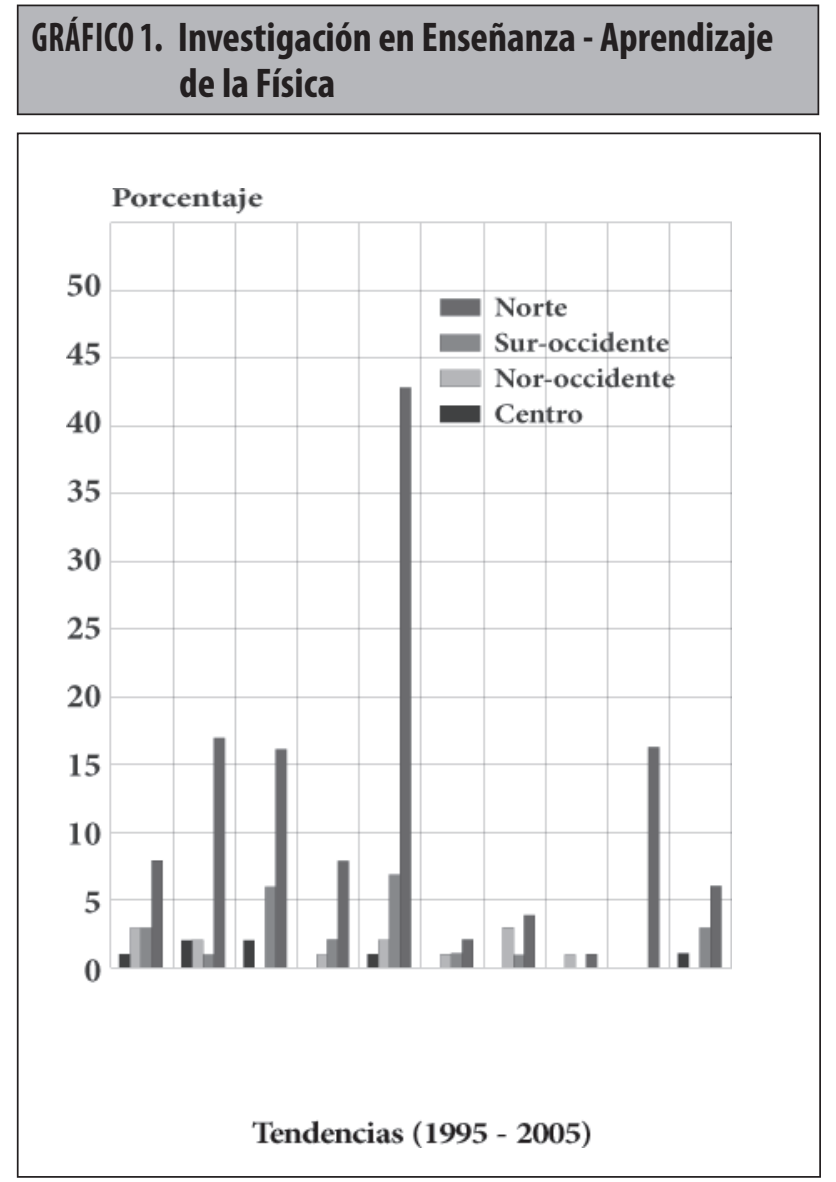
la presentación y organización de los contenidos. Sin embargo, estas investigaciones ponen de manifiesto la dualidad enseñanza-aprendizaje de la física en formas no muy claras ya que, en algunas ocasiones, el interés se centra en uno de los dos aspectos dejando la pregunta sobre la pertinencia de seguir manteniendo la dualidad o asumir uno de sus dos elementos.

\section{Investigación en educación en la física en Bogotá: el caso de IDEP, CIUP la UD}

En el caso de Bogotá se referencian los trabajos de investigación del IDEP, CIUP-UPN, y la Universidad Distrital Francisco José de Caldas, como principales exponentes del campo de la educación en ciencias así como en educación en la física.

\section{Investigaciones sobre aspectos generales de la física y la enseñanza}

El 38\% de los trabajos no centran su interés en la generación de estrategias específicas de enseñanza, sino que buscan reconocer la validez de estudios sobre la ciencia de corte histórico y epistemológico que no necesariamente se compromete con el aprendizaje, dentro de este grupo también se hace énfasis en el desarrollo de conceptos de la física desde diversas perspectivas. Las investigaciones son:

- El principio de conservación del impulso; llevado a cabo por el profesor Fabio Vélez en la categoría de elaboración de conceptos científicos. Aquí se analiza la historia del concepto de la comunicación del movimiento vivenciada en la discusión científica y metafísica del mismo, y evidenciada en los principios pedagógicos de la ciencia en particular de la física. El trabajo muestra los rasgos más persistentes de la edad media, como lo son las creencias religiosas incluidas en los pensamientos científicos, esencialmente en la idea de conservación propuesta por las tendencias cartesianas. Así mismo, el diálogo o los puntos de vista expuestos fueron de: Descartes, Leibniz, Malebranche, Mariotte, Carré y Huygens. En este sentido, la investigación tiene como base que todo fenómeno se debe al desplazamiento de unas partes a otras, lo que implica que toda fuerza se crea por una opuesta a esta, es decir, una fuerza de movimiento crea una de reposo. Así mismo, la fuerza permite explicar los fenómenos físicos teniendo en cuenta cantidades fundamentales como cantidad de materia -masa-, cantidades relativas -vectoriales- y absolutas -escalares-. Obedeciendo una forma constructiva de reglas que no son causas, enunciando que todo principio conlleva una generalidad, lo que indica a la formulación de las teorías como fuente de conocimiento utilizando como herramienta lo que es observable. Sin embargo, y en consideración a los planteamiento de Leibniz, esta forma de concebir el fenómeno podría no estar acorde necesariamente con la experiencia, lo que significa tener en cuenta postulaciones netamente lógicas o con las verdades únicas; tal como lo permitirían las matemáticas postulando la explicación mecánica como herramienta de todo aquello que es entendible. El enfoque del trabajo investigativo permitió formular algunos elementos que están a la base del Principio De La Conservación Del Impulso Y Las Leyes De La Comunicación Del Movimiento En El Siglo XVII tales como: a la cantidad de movimiento como fuerza utilizando cantidades física relativas, la cantidad de movimiento como consecuencia de la parte extensa y la rapidez de los cuerpos, y c. La fuerza como entidad que nomina y hace inteligible a los fenómenos físicos.

- En el otro trabajo del profesor Vélez (El problema de las fuerzas centrífugas: investigación histórica) una de las preguntas centrales es ¿cómo entender la idea de movimiento relativo a partir de los postulados de E. Mach, en su célebre tratado de mecánica (1983), al discutir el problema de las fuerzas centrífugas? Esta investigación asume que E. Mach sólo encuentra una explicación a esta pregunta: el movimiento es necesariamente relativo, las fuerzas centrifugas se deben en consecuencia al movimiento de rotación del cuerpo, no con respecto a la materia presente y en último tér- 
mino al resto de la materia del universo. En este sentido la investigación pretende ahondar en el análisis de una interpretación de la teoría general de la relatividad que considere la aproximación conceptual que deviene del estudio histórico que le es inherente.

- Propuesta didáctica para la introducción a la física de procesos de José González Flores, Clara Inés Chaparro y Juan Carlos Orozco, plantea otra perspectiva de aproximación tanto a la comprensión de los fenómenos físicos como a su enseñanza. En este sentido, se pretende abonar a la discusión sobre preguntas transversales en las que se cuestiona por ejemplo: ¿cómo superar los saberes fragmentados?, ¿cómo superar los saberes generalizados? y ¿cómo abordar la construcción del conocimiento sobre la naturaleza? La aproximación fenomenológica del conocimiento y la enseñanza de las ciencias con base en dos ejes fundamentales de reflexión: las actitudes y la cultura, resultan ser relevantes en este proyecto. De igual forma, se anota que la enseñanza de las ciencias como actividad cultural se caracteriza por ciertas particularidades en diferentes espacios. Se trabaja con categorías explicativas de los profesores de física en formación inicial -estudiantes de la Universidad Pedagógica Nacional de especialización en docencia de las ciencias para el nivel básicocon el propósito de hacer una aproximación a un modelo didáctico que posibilite la construcción de explicaciones a fenómenos del mundo físico por parte de los sujetos involucrados.

- El proyecto Historia y epistemología de las ciencias y la transformación de las practicas de la enseñanza de la ciencia: análisis de procesos, de Germán Bautista discute en forma general la aproximación de la enseñanza de la física, entre otras ciencias experimentales, desde la consideración de los aportes que los análisis históricos y epistemológicos pueden permitir, especialmente desde la perspectiva histórico crítica.

- Dos proyectos en este grupo son: Los problemas de conocimiento, una perspectiva compleja para la enseñanza de las ciencias y los problemas de conocimiento: una estrategia pedagógica y Didáctica para la enseñanza de las ciencias en los niveles básicos (primera fase) de Steiner Edgar Valencia Vargas, Olaga Mercedes Méndez, Juan Carlos Orozco Cruz y Gladys Hermencia Jiménez, Gómez, se encargan de proponer una análisis de la perspectiva de enseñanza problémica desde una perspectiva compleja que la trasciende al punto de permitir reconocer la categoría de problemas de conocimiento como la más adecuada en los procesos de intervención didáctica en la enseñanza de las ciencias. Para el caso de la física aquí surgen alternativas de propuestas vía unidades didácticas de temas relacionados con su enseñanza en el nivel de la primaria. ${ }^{2}$

\section{Investigaciones sobre resolución de problemas físico-matemáticos}

El 19\% de los trabajos manifiesta un enfoque que usa la resolución de problemas buscando enfrentar al estudiante a una determinada situación, involucrando la relación físico-matemática. Este caso reúne las investigaciones realizadas por el profesor Julián Urrea, que aunque toman en cuenta las temáticas directamente asociadas a la física, no se centran en buscar una estrategia de enseñanza de la misma, sino de "matematizar" los aspectos físicos, es decir, de soportar los conceptos de la física basándose principalmente de las matemáticas.

- Las matemáticas en su relación con la física, una propuesta didáctica dentro del nuevo plan de estudios de licenciatura en física de Julián Urrea Beltrán y Mauricio Mendivelso V. propone un análisis de la formación de profesores de física desde la perspectiva del desarrollo de pensamiento matemático y físico. Sus preguntas fundamentales son: “ ¿en qué sentido y dentro de que parámetros los procesos de enseñanza y de comprensión de la física dependen de la eficacia de los métodos propios de pensamiento matemático?", de igual forma en esta investigación se acude al análisis del

2 Revista NODOS Y NUDOS VOL 3 No 212006 
conocimiento matemático que sobre la simetría se puede relacionar con los avances de la física en el siglo XX, siendo característica aquí la postura sobre la relación intrínseca entre física y matemáticas de suerte que la primera depende de la segunda en cuanto su nivel de certeza y exactitud. De esta forma la propuesta concreta en el campo de la formación de maestros radica en "consolidar el papel de las matemáticas en el proceso de enseñanza de la física y propiciar el desarrollo de recontracción de conceptos y de teorías de la física por parte de profesores y estudiantes de los programas de formación de docentes, que tengan relación con las nuevas tendencias de la pedagogía y con los procesos de descubrimiento y de verificación de la ciencia moderna".

- Otro proyecto en esta misma línea es "La relación física-matemática como eje conceptual de la cualificación de docentes y de la innovación curricular en física para la enseñanza en los niveles básico y medio" de Julián Urrea. Aquí se organiza un estudio de corte conceptual en el que se parte de la idea de que la relación física- matemática debe ser experimentada por los futuros profesores a lo largo de su proceso de formación, de suerte que esto pueda servir como referente cuando los profesores ya se encuentren en ejercicio docente y también aborden esta perspectiva de enseñanza. Para el investigador: "En síntesis, se trata de una investigación participativa de tipo didáctico, referida a la enseñanza de la física, en que se propone como estrategia básica el recurso al lenguaje formal de la matemática en los procesos de descripción cuantitativa de los fenómenos y de construcción de teorías acerca del comportamiento de los sistemas físicos. El modelo metodológico descriptivo se define en términos del conocimiento del objeto como una abstracción que conduce a su representación por parte del sujeto, dentro de un esquema descriptivo y explicativo que está en relación, no sólo con la realidad externa, la naturaleza, sino con un conjunto de experiencias, de teorías y de instituciones realizadas alrededor de una problemática planteada desde la pedagogía y la ciencia modernas."

\section{Investigaciones que involucran las ideas previas}

Con un $14 \%$ se encuentran las investigaciones acerca del tratamiento, uso y análisis de las ideas previas, que buscan establecer una estrategia de aprendizaje tomando como punto de partida las ideas que tienen los niños frente a algún concepto en particular entorno a experiencias del estudiante. En este caso las investigaciones encontradas son:

- De las observaciones a las explicaciones: desarrollo del razonamiento sobre hipótesis evidencia en el aprendizaje del concepto de presión de Fernando Sarmiento, en el cual se plantean diversos mecanismos como son: la identificación de las ideas previas presentes en los niños, la innovación pedagógica para abordar dichas ideas erradas o desviadas, y el planteamiento de un nuevo discurso por parte del docente para ser implementado en aras de mejorar el aprendizaje del concepto. La investigación cuestiona la situación de la enseñanza de las ciencias naturales en el Instituto Pedagógico Arturo Ramírez Montúfar (IPARM) de la Universidad Nacional de Colombia al compararla con otras instituciones educativas colombianas desde el presupuesto que: "Los estudiantes aprenden con entusiasmo y motivación conceptos y "datos" científicos en áreas como la física, la biología, la química y la ecología; en muchas ocasiones, se sorprenden ante fenómenos que les son demostrados en el aula; en las salidas de campo observan con avidez su entorno, se formulan preguntas y se arriesgan a proponer explicaciones." Y propone la promoción en el aula del pensamiento y razonamiento científicos, en el contexto del aprendizaje de la física y en especial, del concepto de presión. En particular, esta investigación plantea “...diseñar una serie coherente de ambientes de aprendizaje, constituidos por tareas experimentales y estrategias específicas de intervención docente, que permitieran a estudiantes de segundo a noveno grado de educación básica, desarrollar formas de pensamiento científicas sobre la relación entre hipótesis, evidencia y explicaciones, consistentes con sus niveles de 
desarrollo cognitivo y su conocimiento previo."

- Estudio comparativo entre el uso del software educativo y la manipulación de prototipos como facilitadores del proceso enseñanza aprendizaje de conceptos involucrados en la producción de energía eléctrica por medio del uso de auto generadores de Plamen Nechev, Alberto Bejarano, Paulina Quintero y Sandra Forero. De acuerdo con la información encontrada este proyecto manifiesta explicitamente la importancia de considerar los preconceptos de los estudiantes sobre la energía y sus formas de producción. Argumento que es utilizado en la formulacion de propuestas didácticas que tienen que ver con la generacion de energia en modalidades convencionales o alternativas.

\section{Investigaciones sobre procesos de formalización}

El 14\% muestra las investigaciones que se desarrollan dentro del ámbito cultural, las cuales relacionan la incidencia de los saberes científicos, la actitud y la cultura y la diversidad de un mismo conocimiento en diferentes espacios, dentro de los cuales se pueden nombrar las investigaciones que tienen como fundamento la cultura y las actitudes de los participantes, en el proceso de aprendizaje de cualquier fenómeno; este también es el caso de los trabajos que se basan en los saberes científicos. Los trabajos son:

- Los procesos de formalización y el papel de la experiencia en la construcción del conocimiento sobre los fenómenos físicos de José Francisco Malagón Sánchez, María Mercedes Ayala y Marina Garzón Barrios, del grupo Física y Cultura de la UPN. Esta investigación propone analizar la relación entre las ideas de formalización en la física considerando que "la formalización de los fenómenos físicos involucra dos clases de procesos estrechamente relacionados, que para efectos de análisis se pueden diferenciar: los procesos de mate matización y los procesos de organización de la experiencia y construcción de fenomeno- logías". En este sentido, se cuestiona lo que tradicionalmente se asume como formalizar desde la perspectiva esquematizadora sin que medie lo que los sujetos mismos han venido configurando acerca de la experiencia; es decir, maneras particulares de organizar las representaciones del mundo en las que la formalización se constituiría en un proceso cognitivo como tal y no meramente una forma de matematizar conceptos en este sentido se encuentran los trabajos que se mencionan a continuación.

- La formalización de los fenómenos mecánicos y su relación con la experiencia. análisis histórico-críticos y elementos para propuestas, de Ángel Enrique Romero Chacón, Germán Hernando Bautista Romero, Isabel Garzón Barragán y Luz Dary Rodríguez Rodríguez. Esta investigación forma parte del grupo Física y Cultura de la UPN y se concatena con la línea de trabajo sobre la formalización en física. Particularmente en este análisis se reconoce la importancia de la enseñanza de la mecánica como objeto de estudio, pero desde la perspectiva de procesos de formalización en relación con la experiencia. Su aporte, entre otros, radica en la posibilidad de seguir configurando el abordaje histórico y epistemológico de la física como alternativa en el campo de la investigación en educación en la física, así como en el desarrollo de propuestas concretas alrededor de la enseñanza de la mecánica.

- De la mecánica a la actividad de organizar los fenómenos mecánicos: hacia la construcción de propuestas alternativas para la enseñanza de la mecánica de María Mercedes Ayala, Francisco Malagón, Isabel Garzón, Luz Dary Rodríguez, Juan Carlos Castillo, María Garzón, Eduardo Bautista, del grupo Física y Cultura UPN. Son dos propósitos fundamentales que esta investigación tiene: por un lado caracterizar las maneras particulares en las que la organización de los fenómenos se constituyen en elementos teóricos, a partir del análisis del trabajo de Lagrange sobre la hidrostática y el de Maxwell, sobre las condiciones de equilibrio de los medios elásticos. Y, por otro lado, 
se pregunta por las implicaciones de recontextualizar esta problemática a partir de la selección de problemas así como de la creación de situaciones problémicas en las que tanto el sentido común como las ideas de la física se pongan en juego. De esta manera, la investigación asume por convicción, que cuando se organizan fenómenos no mecánicos se puede estar haciendo este proceso a partir de lo que allí se denomina como las "formas de organización de los fenómenos mecánicos" que no necesariamente les son coherentes en relación con las diferentes perspectivas en las que históricamente fueron validadas -como por ejemplo el caso de la visión de campos en el electromagnetismo-.

\section{Investigaciones desde la perspectiva fenomenológica y de los sistemas dinámicos}

Las investigaciones que validan la perspectiva fenomenológica y los sistemas dinámicos, también reconocen el papel de la recontextualización de saberes como alternativas en la generación de propuestas didácticas en las que se consideran factores culturales asociados a la producción de saberes. Los trabajos son:

- La termodinámica fenomenológica: orígenes conceptuales y elementos de una propuesta pedagógica de María Mercedes Ayala, Ángel Romero, Francisco Malagón, y Edwin García, del grupo Física y Cultura UPN. En este trabajo la pregunta principal es “ $¿$ Cómo replantear la manera en que el estudio de los fenómenos térmicos es abordado en las prácticas de enseñanza, de modo que el estudiante pueda elaborar un sistema conceptual coherente de interpretación de los procesos naturales en el cual la entropía sea un concepto fundamental?".

- Los sistemas dinámicos: una perspectiva contemporánea para la enseñanza de la física, de Clara Inés Chaparro, Rosa Inés Pedreros, Jorge Ignácio Vallejo, Lucia Velasco y Primitivo Acosta. De acuerdo con la información consultada: "El proyecto pretende consolidar una alternativa pedagógica y didáctica, para la enseñanza de la física, que supere los múltiples problemas de la actual forma de enseñanza de la física en el nivel de educación básica y universitaria. Para esto es necesario implementar propuestas de formación de los futuros licenciados en física, como primera fase de la propuesta, para luego en una etapa posterior, realizar una propuesta de formación permanente de los docentes de física en ejercicio. El marco conceptual que acompaña el presente trabajo, aun cuando tiene que ver con una compleja red de conceptos y teorías de corte físico- matemático y pedagógico, se centra en lo que soporta la búsqueda de respuestas a los interrogantes que estructura nuestro problema de investigación. En este sentido hacemos mención a los sistemas dinámicos, la evolución de nuestro problema desde lo que denominamos física de procesos, las estrategias pedagógicas y dos aspectos que resultan de gran interés epistemológico: la distinción entre información y conocimiento y la estructura de las teorías científicas".

\section{Investigaciones sobre la experimentación y el software}

El 10\% muestra las investigaciones que involucran actividades experimentales, dentro de estas investigaciones se pueden mencionar las que buscan innovar en la educación implementando herramientas tecnológicas e involucrando las prácticas de laboratorio y tratamiento de datos como mecanismo de aprendizaje haciendo uso de la informática. Los trabajos son:

- Conozcamos, analicemos y construyamos máquinas de Elizabeth Colmenares, Patricia Moreno y Martha Villareal. Es un proyecto desarrollado como parte de procesos de cualificación de los "proyectos del aula en los CED Nueva Esperanza y CED Luis López de Mesa, durante 2000 y 2001, sobre máquinas en 7 y 4 grado se puede notar que los estudiantes no hacían relaciones entre las partes de la máquina y su funcionalidad en el diseño de modelos experimentales relacionados con máquinas simples, por esta razón, el problema que se abordó es el bajo desempeño en la compe- 
tencia, para argumentar y plantear tipos de irregularidades que presentan los niños de 4 y 6 grado del CED Nueva Esperanza, CED Luis López de Mesa, aspecto que vivencia en la dificultad que tiene los alumnos para realizar acciones como plantear relaciones condicionales, predecir resultados, elaborar conclusiones y realizar diseños experimentales, en este caso con la problemática de máquinas simples". Esta investigación del IDEP fue una de las tres de la década en estudio, específicamente en el campo de la enseñanza de la física desarrollada por profesores de una institución educativa que tienen como referente tanto la vivencia diaria de las problemáticas de enseñanza, como una perspectiva innovadora en la misma. Así, se manifiesta en el contenido del documento consultado que el trabajo, asume la mirada constructivista desde una perspectiva de desarrollo humano y no necesariamente de orden cognitivista, pues para las investigadoras es "el modelo parte de la teoría de cambio conceptual, metodológico y actitudinal" a partir de la generación de problemas que los propios estudiantes vayan identificando y decidiendo acerca de su validez y, en este sentido, la manera como se aborda la problemática también tiene en cuenta las decisiones de los estudiantes.

- Estudio comparativo entre el uso del software educativo y la manipulación de prototipos como facilitadores del proceso enseñanza aprendizaje de conceptos involucrados en la producción de energía eléctrica por medio del uso de autogeneradores de Plamen Nechev, Alberto Bejarano, Paulina Quintero y Sandra Forero. De acuerdo con la información encontrada: "El proyecto pretende comparar la asimilación de conceptos físicos, químicos, mecánicos y electrónicos realizada en estudiantes de grado noveno que manipulan prototipos los tecnológicos a escala frente a aquellos que manejan un software educativo, aprovechando los preconceptos que estos han construido alrededor de la energía y su producción. Vale aclarar que este proceso estará articulado en el marco de la comprensión de la producción de la energía eléctrica y las ven- tajas que el aprovechamiento de las energías alternativas, especialmente la eólica, presentan para tal fin". "El material didáctico... está conformado por un tutorial interactivo (software) y el prototipo funcional a escala que permita, dado su diseño modular ser armado y desarmado a voluntad, materializándose en un kit a ser usado por otros maestros en contextos distintos tras finalizar el proyecto. Este material tiene por tema fundamental la producción de energía eléctrica por medio de un aerogenerador a escala, de manera que los mismos conceptos involucrados en el diseño y construcción del prototipo estén presentes en el software".

- Innovaciones pedagógicas para los laboratorios de física y ciencias naturales, orientadas a la educación media vocacional colombiana a través de la informática educativa de Edgar Manolo Benavides Flores, Analida Atalagracia Hernández Pinchada, Iván Darío Urrea Ospina, Plamen Neichev Nechev, Fabio Wilches y César Mondragón. Se propone plantear un modelo alternativo para educar al alumno en el diseño, ejecución y análisis de los datos obtenidos durante un laboratorio. Relacionando los planteamientos teóricos, las competencias en física y ciencias naturales desde las clases de laboratorio. Se menciona a un grupo de estudiantes que participó en ciertas indagaciones pedagógicas relacionadas con el trabajo experimental inherente al curso de biotecnología, pero no se abordan como fundamento de la investigación. $\mathrm{Al}$ realizar los cursillos, se busca que el docente modifique su metodología de clase, para con eso innovar en el proceso educativo. Las nuevas herramientas ${ }^{3}$-en este caso particular, los productos adquiridos de Nacional Instruments y la Híper guía actualizable de laboratorio, elaborada por el equipo de la innovación-, no solamente cambian loshábitos de trabajo, sino impulsan otros tipos

3 "A partir de una interfaces estándar, consistente en los hardware y software proporcionados por el kit básico educativo de National Instruments ${ }^{\circledR}$, se plantea una metodología para educar al alumno en el diseño, ejecución y análisis de los datos obtenidos durante un laboratorio así como presentar los resultados finales de la experiencia mediante el computador." 
de pensamiento y por ende, tocaron de manera muy sensible las competencias en ciencias, desde un punto de vista de las experiencias que se dan en los laboratorios escolares. Respecto a la planeación de las clases, se busca educar a un grupo de maestros del IPN en la nueva metodología de trabajo en los laboratorios de física y ciencias naturales. En el proyecto se llevó a cabo en su inicio un cursillo o taller, donde los docentes involucrados en la innovación deberían obtener sus propios conocimientos respecto del software y hardware específicos. Se optó por la instalación directa por parte del IPN, de las tarjetas de adquisición. En estas circunstancias, el trabajo pedagógico debe realizarse completamente durante el desarrollo de las clases habituales en el colegio. En forma consecutiva se plantearon las indagaciones en el aula de clases y las respectivas evaluaciones. De modo posterior, se agregó otro compromiso: el diseño y puesta en marcha del software Híper guía actualizable de laboratorio. Las fases fueron: a) diseñar la organización y ejecución de laboratorios por computador, en el cual se ofrecen tareas presentadas como situaciones novedosas para los alumnos y sin indicadores directos de cómo resolverlas. Como actividades específicas se desarrollaron talleres con los docentes involucrados en el proyecto, $b$ ) diseñar y poner en marcha un software denominado Híper guía actualizada de laboratorio y c) participación en cursos y talleres, y otros eventos.

\section{Investigaciones sobre las creencias de los estudiantes y los profesores de física}

Entre los múltiples trabajos que existen hoy en día alrededor de la enseñanza de la física, muchas de las preocupaciones más importantes se basan en el fomento de estrategias apropiadas para mejorar los procesos didácticos en el aula. Así mismo, existen otras tendencias para investigar la influencia de las ideas de los estudiantes a la hora de resolver problemas en el contexto de la enseñanza de la física. Sin embargo, la preocupación por la influencia del pensamiento del profesor en el desarrollo de este tipo de propuestas es algo relativamente nuevo y, podría permitir reconfigurar muchas de las investigaciones del carácter anteriormente mencionado.

Investigar el pensamiento del profesor de física se constituye en un reto contemporáneo en Colombia, especialmente porque cuestiona aquellos fundamentos epistemológicos en los que su conocimiento tiene sentido histórico, político y social. En efecto, si se considera que el profesor de física, desde su intencionalidad como formador de sujetos, ha construido un conocimiento de contenido que le es propio, entonces la forma como el profesor se posiciona ante la educacion en ciencias puede implicar la construcción de nuevas categorías de formación de futuros profesores de física, así como de relativizar el conjunto de propuestas didácticas que se ponen en juego a la hora de enseñar.

En la medida en que se reconoce históricamente un debate sobre la pertinencia, necesidad y validez del estudio de pensamiento del profesor que regula sus practicas, se destaca con esto, de la psicología cognitiva, que el pensamiento es causa de la acción, por tanto la practica del maestro puede cambiar si se cambia su pensamiento. Entonces, la labor investigativa se desvía, dejando de un lado la búsqueda por la constitución de nociones universales de profesor, de maestro, de docente de física, y en lugar de ello, se pregunta por el conocimiento de contenido del profesor; lo que se llamó conocimiento profesional del docente. Así mismo, la pregunta por la epistemología también tiene sentido en esta reflexión, ya que generalmente se ha considerado estudiar como aplicar la epistemología de la ciencia al conocimiento del profesional para ver si se puede validar o no. De tal suerte que siempre se mira el conocimiento del profesor en escalones inferiores que no alcanzan los peldaños superiores, no alcanzan a ser científicos, y se catalogan solo como creencias o imágenes. De todo esto surge, también, la importancia de hablar de una epistemología específica del conocimiento del profesor de física. Los proyectos encontrados en esta linea son:

- Creencias y posibilidades de cambio epistemológico en profesores de física de educación media, El caso del concepto de movimiento, 
de Gerardo Andrés Perafán Echeverri, Jaime Duván Reyes Roncancio, Jurin Janey Burbano Muñoz y Janett Barbosa Urbano. El proyecto hace parte de los desarrollos de la línea de investigación: teorías y prácticas del profesor, la cual se encuentra adscrita a la nueva propuesta curricular de la Maestría en Educación que ofrece la Facultad de Educación de la UPN. Igualmente, constituye un punto de continuidad con los trabajos que se vienen desarrollando en la línea de epistemología y pedagogía desde el énfasis de evaluación escolar de la mencionada maestría. Este proyecto se inscribe, fundamentalmente, dentro de la línea de epistemología y conocimiento del profesor, que constituye uno de los ejes centrales del trabajo que adelanta el grupo. En este estudio de caso las preguntas que delimitan la indagación son: ¡cuál es la naturaleza, tipo y función de los referentes epistemológicos que mantienen algunos profesores de física?, ¿es posible favorecer un cambio en las creencias epistemológicas de los profesores de física?, ¿influye el cambio de las creencias epistemológicas de los profesores en la enseñanza de conceptos?

- El conocimiento de contenido del profesor de física sobre las nociones de fuerza y de espacio. Estudios de caso, de Gerardo Andrés Perafán, Jaime Duván Reyes, Janeth Barbosa y Jurin Burbano, se incribe dentro de la línea de epistemología y conocimiento del profesor, que constituye uno de los ejes centrales del trabajo que adelanta el grupo Invaucol con el desarrollo de sus anteriores investigaciones. El programa de investigación contempla varias etapas: en la primera se estudió lo relacionado con las creencias y posibilidades de cambio epistemológico en estudiantes de física de décimo grado. Esta etapa tuvo una duración aproximada de nueve meses. En la segunda etapa se estudió lo relacionado con las creencias y posibilidades de cambio epistemológico en profesores de física, de décimo grado, en lo que tiene que ver con la caracterización de los referentes epistemológicos sobre la categoría de movimiento en los docentes. Con duración de doce meses. En la tercera y cuarta etapa que se propone para los años 2005 y 2006, se estudiará el conjunto de tales referentes en las categorías de fuerza y de espacio, respectivamente. En este estudio de caso las preguntas que delimitan la indagación son: ¿cuál es el conocimiento de contenido del profesor de física en las categorías de fuerza y de espacio?, ¿cuál es la naturaleza, tipo y función de los referentes epistemológicos que mantienen algunos profesores de física?, ¿cuál es carácter del conocimiento que le es inherente al docente de física como sujeto social y como formador de sujetos? El rango de validez de los resultados presentados en este trabajo estará referido a los contextos de aula de los profesores de física. Su pertinencia está delimitada por el interés de la comunidad académica internacional orientado a conocer qué y cómo piensan los profesores de su actividad académica, qué sentido le atribuyen a la misma y cómo la naturaleza de ese pensamiento determina la eficacia de las acciones de enseñanza y, de otra parte, caracterizan el conocimiento del profesor como un conocimiento complejo, polifónico y dialógico, en tanto que sus discursos implícitos en las practicas develen esa misma multiplicidad de determinaciones históricas que construyen un conocimiento particular: el del profesor.

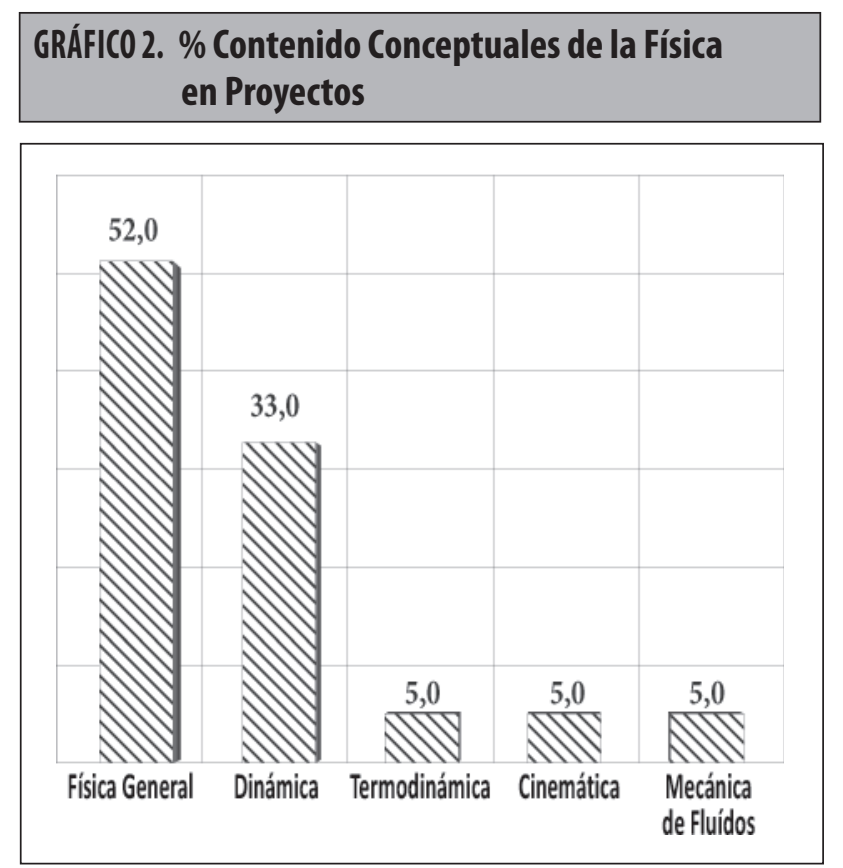




\section{Temáticas de la física que son abordadas en los trabajos del IDEP y el CIUP}

Aquí se destacan las investigaciones que trabajan temáticas específicas de la física, tales como cinemática, termodinámica, dinámica, entre otras. Esta clasificación busca mostrar el impacto de los contenidos de la física que se desarrollan en los trabajos realizados por los institutos estudiados.

Llama la atención que el $52 \%$ no se compromete con un campo conceptual específico de la física dejando un margen bastante amplio en sus estudios. De otra parte, el 33\% tienen como eje los conceptos de fuerza e impulso, los cuales hacen parte de la dinámica. Por último con un $5 \%$ se encuentran investigaciones dirigidas a temáticas como termodinámica, mecánica de fluidos y cinemática.

\section{Investigaciones en educación en física en la Universidad Distrital Francisco José de Caldas}

Las investigaciones en enseñanza de la física en la Universidad Distrital Francisco José de Caldas se pueden distinguir bajo la categoría de propuestas de transformación pedagógica de la escuela y la enseñanza de la ciencia y la tecnología. Principalmente se destacan los trabajos del profesor Hurtado Alejandro (Diseño, construcción e implementación de material didáctico en física - Física con Interactive Physics) y su grupo de investigación (Fisinfor, conformado por Alejandro Hurtado, Oscar Ocaña y Carlos Lombana) que utilizan software en el campo de la enseñanza de la física como alternativa didáctica. Uno de los trabajos es el titulado "Integración del experimento tradicional, el experimento computarizado y la simulación como propuesta alternativa para el aprendizaje y enseñanza de la física" este trabajo involucra: "El estudio de los fenómenos en las condiciones propias del aula y de los laboratorios donde se destacan o se separan los mismos de la naturaleza circundante constituye un arma valiosa del poderoso método experimental en la Enseñanza de la Física”. Aquí el interés fundamental parece ser la eficiente utilización del dispositivo por parte de los usuarios de manera que la física se haga más exequible por parte de los estudiantes. En este caso también se encuentran trabajos que propenden por el diseño y construcción de herramientas didácticas que utilizan software especializado.

En este sentido, las investigaciones de este campo se fundamentan en la posibilidad de transformar las prácticas educativas del profesor de física que tradicionalmente no utilizan software para el caso específico de fenómenos como la atracción gravitacional. Al parecer, la apuesta por el computador no se da desde el instrumento como tal sino en correspondencia con una manera de cualificar los procesos de modelación de la realidad; aspecto que destaca el grupo Fisinfor como fundamental en sus investigaciones respecto de la contribución que en este campo se hace a la formación de los estudiantes de licenciatura en física.

\section{Comentarios finales}

La búsqueda por las huellas de la investigación educativa en la física durante la década 1995-2005 permite vislumbrar las tendencias que los grupos e investigadores desarrollaron y siguen fortaleciendo, estas se pueden considerar mas de corte didáctico que pedagógico, pues a diferencia de los resultados expuestos por Hernández (2002), en el caso de los trabajos aquí comentados se aprecian compromisos expedíos de los investigadores por dar cuenta de procesos de mejoramiento o análisis de aprendizaje de conceptos físicos concretos.

Aún cuando los trabajos comentados no se revelan explícitamente en el campo del conocimiento escolar, algunos de estos sí muestran interés por el conocimiento de los profesores alrededor de los conceptos físicos o de propuestas didácticas para su enseñanza. Esta situación, aunada a los trabajos que se enfocan en el conocimiento del profesor se manifiestan primigeniamente en esta década para el caso del estudio del conocimiento profesional del profesor de física en Bogotá, lo cual permite afirmar que son un antecedente importante en la manera como se va configurando esta perspectiva investigativa que hoy en día ha venido trascendiendo a campos como el conocimiento pedagógico del contenido o en conocimiento didáctico del contenido. 
Puede también afirmarse que la fuerte tendencia a seguir analizando aspectos generales de la física y su enseñanza, estaría en algún grado relacionado con la falta de perspectivas concretas a nivel didáctico desde las cuales los investigadores puedan asentarse y comenzar a validar con mayor sentido la idea de la investigación de un conocimiento escolar, con contenido que trasciende lo pedagógico general.

Si bien, el perfil de las investigaciones desde perspectivas fenomenológicas y las de procesos de formalización tienen el sesgo de una mirada alternativa a la investigación educativa en la física, su interés sigue centrado en ahondar el conocimiento de base físico y no necesariamente propenden por una confluencia o "amalgama" en términos de Shulman (1984) entre conocimiento pedagógico y conocimiento de la física, que derive en una propuesta concreta tanto de investigación como de formación docente. En este mismo sentido, se podrían relacionar aquí las investigaciones sobre resolución de problemas físico-matemáticos y aquellas sobre la experimentación y el software, dado que se configuran como posibilitadores de reflexión de contenidos concretos y su enseñanza pero desde un enfoque didáctico poco concreto, pues más bien tienen en cuenta presupuestos que usualmente se ubicarían desde la pedagogía como referente teórico o desde la didáctica general, al parecer acéfala de perspectiva teórico investigativa.

\section{Bibliografía}

Ayala, M. et al. (2001). De La Mecánica A La Actividad De Organizar Los Fenómenos Mecánicos: Hacia La Construcción De Propuestas Alternativas Para La Enseñanza De La Mecánica. Informe de investigación: Centro de Investigaciones Universidad Pedagógica Nacional CIUP.

Ayala, M., et al. (1996). La Termodinámica Fenomenológica: Orígenes Conceptuales Y Elementos De Una Propuesta Pedagógica. Informe de investigación: Centro de Investigaciones Universidad Pedagógica Nacional CIUP.
Bautista, G. (1996). Historia Y Epistemología De Las Ciencias y La Transformación De Las Practicas De La Enseñanza De La Ciencia: Análisis De Procesos. Informe de investigación: Centro de Investigaciones Universidad Pedagógica $\mathrm{Na}-$ cional CIUP.

Benavides, E., et al. (2002). Innovaciones Pedagógicas Para Los Laboratorios De Física Y Ciencias Naturales, Orientadas A La Educación Media Vocacional Colombiana A Través De La Informática Educativa. Informe de investigación: Centro de Investigaciones Universidad Pedagógica Nacional CIUP.

Chaparro, C., et al. (2001). Los Sistemas Dinámicos: Una Perspectiva Contemporánea Para La Enseñanza De La Física. Informe de investigación: Centro de Investigaciones Universidad Pedagógica Nacional CIUP.

Colmenares, E. (2002). Conozcamos, Analicemos y Construyamos Máquinas. Instituto para la Investigación Educativa y el Desarrollo Pedagógico del Distrito Capital IDEP. Informe de Investigación.

Flores, G., et al. (1999). Propuesta Didáctica Para La Introducción A La Física De Procesos. Informe de investigación: Centro de Investigaciones Universidad Pedagógica Nacional CIUP.

Gaitán, M. J. (1998). Técnicas de investigación en Comunicación Social. Madrid: Editorial Síntesis.

Hernández, C. A. (2002). Aproximación A Un Estado Del Arte De La Enseñanza De Las Ciencias En Colombia. Universidad Nacional de Colombia...

Hurtado, A., et al. (2000). Diseño, construcción e implementación de material didáctico en física - Física con Interactive Physics. Informe de investigación: Centro De Investigaciones y Desarrollo Científico 'CIDC de la Universidad Distrital Francisco José de Caldas.

Nechev, P., et al. (2002). Estudio Comparativo Entre El Uso Del Software Educativo Y La Manipulación De Prototipos Como Facilitadores Del Proceso Enseñanza Aprendizaje De Conceptos 
Involucrados En La Producción De Energía Eléctrica Por Medio Del Uso De Autogeneradores. Informe de investigación: Centro de Investigaciones Universidad Pedagógica $\mathrm{Na}-$ cional CIUP.

Ortiz, F., et al. (1993). Metodología De La Investigación El Proceso Y Sus Técnicas. Ed Limusa.

Perafan, G. A.; Reyes, J. D., et al. (2003). “Creencias y Posibilidades de Cambio epistemológico en estudiantes de Física de Educación Media. Estudio de Caso". En: Pedagogía y Saberes, U.P.N. (18).

Perafán, G., et al. (2002). Creencias Y Posibilidades De Cambio Epistemológico En Profesores De Física De Educación Media, El caso del Concepto de Movimiento. Informe de investigación: Centro de Investigaciones Universidad Pedagógica Nacional CIUP.

Perafán, G., et al. (2004). El conocimiento de contenido del profesor de física sobre las nociones de fuerza y de espacio. Estudios de caso. Informe de investigación: Centro de Investigaciones Universidad Pedagógica $\mathrm{Na}$ cional CIUP.

Reyes, J. (2007). Análisis Epistemológico Y Cultural De La Investigación En Educación En Ciencias En Colombia 1995 - 2005. Informe final proyecto de investigación Universidad Distrital Francisco José De Caldas. Grupos de investigación: Invaucol-Intercitec. Código: 2403520305

Romero, A., et al. (2003). La Formalización De Los Fenómenos Mecánicos Y Su Relación Con La Experiencia. Análisis Histórico-Críticos Y Elementos Para Propuestas. Informe de investigación: Centro de Investigaciones Universidad Pedagógica Nacional CIUP.

Sarmiento, F. (2001). De Las Observaciones A Las Explicaciones: Desarrollo Del Razonamiento
Sobre Hipótesis Evidencia En El Aprendizaje Del Concepto De Presión. Informe de investigación: Instituto para la Investigación Educativa y el Desarrollo Pedagógico del Distrito Capital IDEP .

Shulman, L. (1984). Paradigmas y Programas de Investigación en el Estudio de la Enseñanza: Una Perspectiva Contemporánea. En: Wittrock, M. C. (comp.) La Investigación de la Enseñanza, I. En foques, teorías y métodos. Paidós

Urrea, J. y Mendivelso, M. (1999). Las Matemáticas En Su Relación Con La Física, Una Propuesta Didáctica Dentro Del Nuevo Plan De Estudios De Licenciatura En Física. Informe de investigación: Centro de Investigaciones Universidad Pedagógica Nacional CIUP.

Urrea, J. (1999). La relación física-matemática como eje conceptual de la cualificación de docentes y de la innovación curricular en física para la enseñanza en los niveles básico y medio. Informe de investigación: Centro de Investigaciones Universidad Pedagógica Nacional CIUP.

Valencia, E., et al. (2001). Los Problemas De Conocimiento, Una Perspectiva Compleja Para La Enseñanza De Las Ciencias y Los Problemas De Conocimiento: Una Estrategia Pedagógica Y Didáctica Para La Enseñanza De Las Ciencias En Los Niveles Básicos (Primera Fase)

Vélez, F. (1999). El Principio De Conservación Del Impulso. Informe de investigación: Centro de Investigaciones Universidad Pedagógica Nacional CIUP.

Vélez, F. (2003). El Problema De Las Fuerzas Centrífugas: Investigación Histórica. Informe de investigación: Centro de Investigaciones Universidad Pedagógica Nacional CIUP. 